\title{
Relato de uma Experiência de Integração Universidade- Governo-Escola na Construção Coletiva de um Sistema de Monitoramento do Programa Um Computador por Aluno
}

\author{
Isabel Fernandes ${ }^{1}$, Ronaldo Goldschmidt ${ }^{1}$, Mônica Norris ${ }^{2}$, Rogério Nunes ${ }^{2}$ \\ ${ }^{1}$ Instituto Multidisciplinar - Universidade Federal Rural do Rio de Janeiro, ${ }^{2}$ Secretaria \\ Municipal de Educação de Piraí \\ projeto.memore@gmail. com
}

\begin{abstract}
Brazilian government has launched its own version of the 'One Laptop per Child' program. Called UCA, this initiative has already provided hundreds of laptops for students and trained several teachers from many schools. However, UCA does not have tools that help managers monitor, understand and evaluate which curricular and extracurricular activities have been developed with the laptops. Designed with financial support from federal government, MEMORE is a computational system that provides management information about the use of laptops in UCA's context. Therefore, this article aims to report the experience of integrating university-government-school in the collaborative task of building this system.
\end{abstract}

Resumo. O governo brasileiro lançou o "Projeto Um Computador por Aluno" (UCA), que vem distribuindo laptops e realizando capacitação dos professores para a utilização de tecnologia computacional no ensino-aprendizagem. Este projeto carece de instrumentos que permitam às instâncias gestoras conhecêlo melhor, acompanhá-lo e avaliá-lo. Para atender à demanda por instrumentos de monitoramento do projeto UCA, o governo federal está investindo no desenvolvimento do MEMORE, um sistema computacional que provê informações gerenciais sobre como os laptops distribuidos têm sido utilizados pelos beneficiários do programa. Diante deste cenário, o presente artigo tem como objetivo relatar a experiência de integração universidadegoverno-escola na tarefa de construção coletiva do referido sistema.

\section{Introdução}

O emprego de tecnologias da informação e comunicação (TIC) na mediação de processos de aprendizagem vem crescendo mundialmente nos últimos anos [Tarja, 2008]. Um exemplo disso, no Brasil, são as iniciativas do governo para implementar ações que incorporem computadores no cotidiano das escolas do país. O Projeto Um Computador por Aluno (UCA) e o Programa Um Computador por Aluno (PROUCA) são duas dessas iniciativas e fazem parte das políticas públicas do governo federal para o desenvolvimento da Educação [Meneses, 2011]. A primeira iniciativa (UCA) implantou laptops de baixo custo em cerca de 300 escolas distribuídas por todo o país e vem promovendo a capacitação de seus docentes para uso da tecnologia. A segunda (PROUCA) consiste de um programa que permite que prefeituras tenham autonomia e facilidades financeiras na compra direta dos laptops. Visando a simplificação do texto, 
as duas iniciativas serão referenciadas indistintamente ao longo deste artigo pela sigla PROUCA.

Somente a distribuição de computadores pelas escolas, assim como a implantação de laboratórios e o treinamento de docentes, não é suficiente para assegurar o uso pedagógico desses recursos de forma plena e satisfatória [Motta et al. 2009]. O PROUCA carece de instrumentos que permitam às instâncias gestoras e de governo conhecer, acompanhar e avaliar ações pedagógicas vinculadas à utilização dos laptops nas salas de aula e fora delas [CNPq, 2010]. Para atender à demanda por instrumentos de monitoramento do PROUCA, o governo federal está investindo no desenvolvimento do MEMORE, um sistema computacional que deverá prover informações gerenciais sobre como os laptops distribuídos têm sido utilizados pelos beneficiários do programa. Espera-se que a partir do uso do MEMORE, gestores educacionais possam encontrar padrões e informações sobre práticas pedagógicas que auxiliem tanto no estudo da complexidade e amplitude do PROUCA quanto na definição de estratégias e políticas educacionais baseadas no uso das TIC.

Diante deste cenário, o presente artigo tem como objetivo apresentar um relato da experiência de integração universidade-governo-escola na construção coletiva do MEMORE. Este relato compreende detalhes sobre a parceria firmada, o fluxo de conhecimento intra/entre as equipes técnica e da área de Educação promovendo situações de aprendizagem para ambas, subprodutos gerados, resultados indiretos, e o detalhamento do conjunto de ações desenvolvidas ao longo das etapas do projeto.

O restante do texto encontra-se estruturado em mais seis seções. A seção 2 descreve um histórico do desenvolvimento do projeto. Detalhes técnicos sobre o MEMORE são expostos na seção 3. As seções 4 e 5 apresentam, respectivamente, a revisão bibliográfica e o conjunto de ações executadas ao longo do processo de desenvolvimento do MEMORE. A seção 6 descreve os resultados indiretos e subprodutos do trabalho. Considerações finais sobre o projeto, assim como perspectivas de ações futuras estão indicadas na seção 7.

\section{O histórico do MEMORE}

O ambiente MEMORE é um projeto multidisciplinar que vem sendo modelado com base em técnicas de Inteligência Artificial [Russell e Norvig, 2004], Mineração de Dados [Goldschmidt e Passos, 2005] e Engenharia de Software [Pádua Filho, 2005]. A sua arquitetura permite a replicação e a implantação do ambiente nas diversas escolas vinculadas ao PROUCA, proporcionado assim uma integração incremental e gradual de informações oriundas de diferentes contextos geográficos. A área fim do projeto é a Educação. Ela permeia todo o projeto e é de suma importância para o MEMORE, pois é a base do domínio de problema estudado [Moraes e Almeida 2011].

A equipe inicial, proponente do MEMORE, contou com a participação de dois professores da área de banco de dados, um de inteligência computacional, um de programação de computadores e um de engenharia de software. Ao todo, foram cinco professores doutores, oriundos de quatro instituições públicas do estado do Rio de Janeiro. Neste grupo, apenas um possuía também algum conhecimento de Educação, resultado de experiência com a aplicação da Computação como ferramenta de suporte às práticas docentes e de ensino-aprendizagem em educação a distância. No entanto, este 
conhecimento estava muito aquém da demanda do projeto. Era insuficiente para apoiar o levantamento das necessidades, em amplitude e profundidade, para se modelar um sistema de informação que pudesse monitorar remotamente a utilização dos laptops fornecidos pelo PROUCA.

Por avaliar o MEMORE como um produto útil ao sistema educacional do estado, o projeto também recebeu apoio do "Centro de Tecnologia da Informação e Comunicação do Estado do Rio de Janeiro (PRODERJ) [...], órgão gestor de Tecnologia da Informação e Comunicação do Governo do Estado [...] e responsável pela política de TIC no âmbito da administração pública estadual"l. Este apoio veio em forma de disponibilização de toda a infraestrutura de hospedagem de servidor, segurança de dados e endereço físico de acesso para que os módulos do MEMORE pudessem operar.

A combinação entre equipe, infraestrutura e ideia inovadora do produto de software proposto certamente contribuiu para que o projeto MEMORE fosse bem avaliado pela comissão responsável pelo edital CNPq a que o projeto foi submetido. Isso resultou na concessão do fomento para que o grupo pudesse dar continuidade ao desenvolvimento do projeto e materializasse a idéia proposta. Assim, no recebimento da carta de outorga, deu-se início a uma busca por parcerias. Era necessária uma escola piloto para o projeto.

Então, desde a aprovação do projeto pelo CNPq, a equipe do projeto MEMORE realizou contato em várias instâncias gestoras, em busca de uma escola no estado do Rio de Janeiro para atuar como laboratório no desenvolvimento e na implantação do ambiente proposto. Até onde foi possível observar, a grande maioria das escolas contatadas ainda se encontrava em fase de implantação dos laptops e de capacitação dos docentes para atuar no PROUCA. Desta forma, não dispunham nem de tempo e nem de experiência suficiente no programa para auxiliar no desenvolvimento do sistema proposto.

Felizmente, no final de março de 2012, a partir de uma indicação da Coordenação do Projeto UCA no estado do Rio de Janeiro, a equipe do Núcleo de Tecnologia Educacional da Secretaria Municipal de Educação de Piraí percebeu o potencial de aplicação e de ajuda que o ambiente MEMORE poderia vir a proporcionar e, mesmo dispondo de pouco tempo em suas atividades diárias, dispôs-se a apoiar formalmente o desenvolvimento do ambiente. A partir desta formalização da parceria, duas escolas foram indicadas para participar como escolas piloto: uma escola do projeto UCA e a outra que recebeu os laptops adquiridos pela prefeitura de Piraí via PROUCA.

A escolha das escolas se deu a partir da compreensão que a equipe da Secretaria Municipal de Educação (SME) teve com relação ao projeto MEMORE. Era necessário escolher uma escola que contasse com gestores e orientadores pedagógicos abertos a projetos inovadores. Outro ponto a ser observado foi o perfil docente. Era necessário um corpo social neofílico que se motivasse com novidades. O fato de uma das escolaspiloto selecionadas possuir um técnico em Computação em tempo integral também foi um ponto favorável na escolha. Estes critérios orientaram a escolha da E.M. Rosa Carelli da Costa. A outra escola, o CIEP 477 Professora Rosa da Conceição Guedes, foi

\footnotetext{
${ }^{1}$ http://www.proderj.rj.gov.br/quem_somos.asp.
} 
selecionada em função de sua experiência no contexto UCA e do critério estabelecido pelo edital do $\mathrm{CNPq}$ que forneceu apoio financeiro ao projeto: o trabalho deveria envolver uma escola contemplada diretamente pelo governo federal via projeto UCA.

\section{O ambiente MEMORE}

O ambiente MEMORE vem sendo modelado com base em técnicas de Inteligência Artificial [Russell e Norvig, 2004], Mineração de Dados [Goldschmidt e Passos, 2005] e Engenharia de Software [Pádua Filho, 2005], cujo funcionamento está distribuído em três módulos principais.

O Módulo I, denominado Coleta de Dados, tem como objetivo capturar informações sobre como cada laptop é utilizado por seu responsável, identificando aplicativos acessados e detalhes de sua utilização (por exemplo, o histórico de websites acessados). A coleta de dados é realizada por um agente de software puramente reativo [Russell \& Norvig, 2004] denominado agente de coleta de dados. Instalado em cada laptop, este agente percebe o momento em que cada software é iniciado e finalizado a fim de registrar a data e a hora do respectivo evento. No encerramento de cada software monitorado, o agente de coleta de dados interage com o usuário do laptop e sempre que a utilização tenha sido para fins acadêmicos, o agente indaga, por exemplo, sobre as disciplinas envolvidas, o tipo de atividade realizada, a forma de trabalho (individual ou em grupo) e o local de utilização do laptop.

Conforme o próprio nome sugere, este módulo II, denominado Transferência de Dados, tem como objetivo transportar os dados coletados em cada laptop para um banco de dados central. A transferência de dados é realizada por um segundo agente de software puramente reativo. Também instalado em cada laptop, este agente monitora o conteúdo de uma pasta previamente especificada. Executa, periodicamente, o processo de envio dos dados coletados para um banco de dados central único (denominado central de análise), sempre que determinadas condições (volume mínimo de informações coletadas e disponibilidade de internet) são atendidas.

O Módulo III, denominado Descoberta de Conhecimento, tem como objetivo permitir consultas, visualizações e análises sobre os dados coletados junto aos laptops. Envolve técnicas de Mineração de Dados [Goldschmidt e Passos, 2005] e de construção de relatórios gerenciais que auxiliem docentes, gestores do PROUCA e de outras instâncias do governo, na análise e interpretação das informações coletadas. Baseia-se na visão pragmática de Goldschmidt e Passos (2005) de que a descoberta de conhecimento é um processo que se subdivide em três grandes etapas: préprocessamento, mineração e pós-processamento.

Maiores detalhes técnicos sobre o MEMORE e o tipo de informação provida pelo ambiente podem ser encontrados em Goldschmidt et al. (2012).

\section{Busca por uma metodologia para a construção coletiva do MEMORE}

Para Santos et al. (2012), dentre os muitos fatores que parecem estar vinculados ao baixo desempenho do ensino básico no Brasil está a carência de recursos da tecnologia digital nas escolas. Quando tais recursos estão disponíveis na escola, em geral, há a falta de habilidade dos professores para utilizá-los. Adicionalmente, estes mesmos autores afirmam que a tarefa de avaliar o ensino e seus resultados, com segurança para registrar 
qualquer afirmação a cerca dos resultados destas avaliações, é uma das questões mais complexas da área da Educação. Trucano (2012) também afirma que o impacto do uso das TIC para a melhoria do ensino nas escolas é de difícil aferição e permanece como um tema aberto ao debate e à pesquisa.

Alinhada com os autores citados acima, Lacerda (2012) afirma que a informática passa a ser indispensável para o aluno buscar conhecimento. Para alcançar resultados efetivos, a autora afirma que é necessário entender como a informática funciona no contexto educacional. É preciso conhecer suas vantagens e também desvantagens.

No contexto dos programas voltados à distribuição de laptops de baixo custo a crianças e jovens nos diversos países do mundo, ainda são raras as iniciativas de investigação dos resultados obtidos [Hansen et al. 2012]. Além disso, a maioria dos estudos existentes foi conduzida em países desenvolvidos [Hansen et al. 2012] e apresenta diferentes abordagens metodológicas com diferentes enfoques, critérios e níveis de rigor científico [Penuel, 2006].

Uma revisão detalhada e recente da literatura sobre a avaliação de programas OLPC pelo mundo pode ser obtida em [Nugroho e Longsdale 2010]. Os autores reportam que tais programas, incluindo o PROUCA brasileiro, concentram suas avaliações em estudos de caso específicos cujas coletas de dados para análise, quando existem, são pontuais e isoladas, inviabilizando um acompanhamento de longo prazo. Em nenhum dos programas retratados por Nugroho e Longsdale (2010) foi possível identificar a existência de ferramentas informatizadas que proporcionassem apoio à captação contínua, ao armazenamento centralizado e à análise de longo prazo sobre os dados acerca da utilização dos computadores fornecidos aos estudantes.

No trabalho de Burd (1999), há uma revisão bastante rica sobre quais marcos referenciais (por exemplo, o construcionismo) os softwares voltados para apoiar a aprendizagem deveriam ser construídos. De forma análoga a Burd (1999), Perry (2005), em seu trabalho, faz um levantamento bastante amplo de várias propostas metodológicas, ciclos de vida e modelos de maturidade, produtos voltados para o desenvolvimento de software educacional. Em seguida propõe uma metodologia. Nela, busca articular o trabalho de três grupos de especialistas: 1) professores, 2) designers e 3) programadores. A proposta busca representar o que de comum apareceu nas metodologias analisadas. Com uma abordagem mais voltada para a visão do engenheiro de produção, a autora propõe tratamento de dois pontos apontados como os mais caros do desenvolvimento de software educacional: ciclo de interações e de avaliação do produto, ambos realizados com o usuário. Propõe que o software vá sendo desenvolvido por meio de prototipagem evolutiva. Cada protótipo gerado engloba funcionalidades elencadas em uma iteração com os usuários. A sugestão é que a metodologia proposta seja utilizada para produzir softwares para apoiar o ensino. No estudo de caso do seu trabalho, a autora mostra que a metodologia trouxe resultados satisfatórios no desenvolvimento de um software a ser aplicado na aprendizagem dos conteúdos de química.

As metodologias propostas por Burd (1999) e por Perry (2005) ficaram aquém das demandas do MEMORE, pois, não fazem nenhuma menção para técnicas de integração e gestão da comunicação em equipes de áreas distintas (área técnica e educação), não abordam técnicas ou melhores práticas para gestão de projetos 
educacionais com grandes equipes, não tratam construção coletiva, não abordam práticas de gestão da integração universidade-governo-escola como é o caso do projeto MEMORE.

Com uma proposta de processo de desenvolvimento de software, o trabalho dos autores Benitti et al. (2005) amplia a proposta anterior [Perry 2005]. Neste processo, os autores chamam a atenção para uma base pedagógica que fundamente sua construção. Mencionam que a falta desta base leva muitos softwares com ideias interessantes a não serem utilizados. O processo de desenvolvimento proposto pelo autor parece ser bastante adequado para softwares que apóiem o ensino, mas não para os softwares de gestão, como é o caso do MEMORE.

Após a revisão da literatura sobre metodologias de desenvolvimento de softwares educacionais, percebeu-se que, dadas as particularidades do projeto MEMORE, ações diferenciadas seriam necessárias. O conjunto dessas ações foi sendo colaborativamente delineado pela equipe multidisciplinar ao longo da execução das etapas do projeto.

\section{Conjunto de ações adotadas no projeto}

Para nortear o desenvolvimento, foi planejado um esboço de organização e ação que, embora precise ser experimentado outras vezes antes de poder ser classificado como uma metodologia, mostrou-se útil no contexto do MEMORE e segue descrito abaixo.

O conjunto de ações adotado nesta parceria universidade-governo-escola para o desenvolvimento do MEMORE compreende sete macro-passos ilustrados na figura 1.

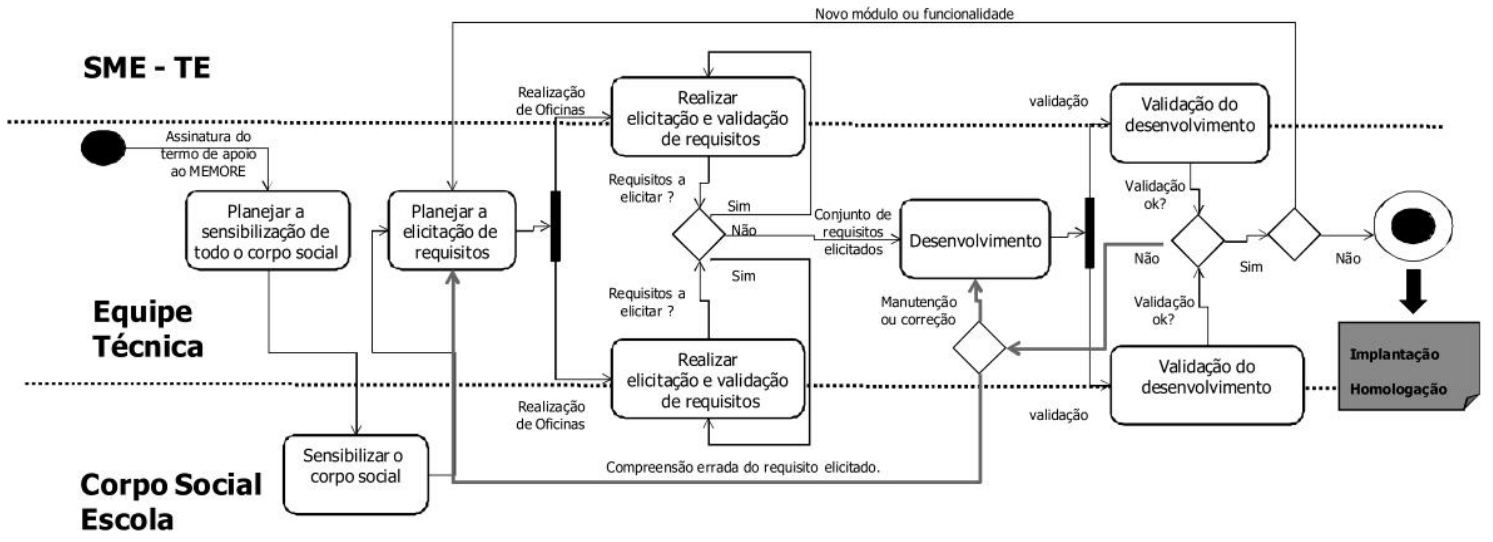

Figura 1. Visão gráfica dos macro-passos adotados no projeto do MEMORE.

Para desenhar a figura da visão gráfica dos macro-passos adotados no projeto MEMORE, foi utilizada a técnica do diagrama de atividades disposta em Pádua Filho (2005). O círculo preto e círculo branco com o interior preto representam as atividades inicial e final do diagrama. Os retângulos de cantos arredondados representam as atividades intermediárias, ou seja, os macro-passos. As setas representam a transição de uma atividade para a outra. Alguns destes fluxos estão nominados com o objetivo de tornar mais claro o evento que promoveu a transição de uma atividade intermediária para a outra. Separadas por retas pontilhadas, as raias de natação servem para delimitar as atividades das quais os grupos da SME, equipe técnica e corpo social da escola participam. 
O fluxo de conhecimento intra/entre as equipes técnica, de pesquisa e educação pode ser classificado como o item mais complexo e desafiador do projeto MEMORE. O grupo envolvido no projeto MEMORE é composto pelas equipes diretivas das duas escolas-piloto, professores e alunos das oito turmas selecionadas das duas escolas para o estudo de caso do projeto, seis alunos de iniciação científica, três desenvolvedores profissionais e cinco professores pesquisadores, sendo esses últimos todos da área da Computação. Ou seja, são muitas pessoas que precisam se manter atualizadas com todas as informações sobre o que ocorre ao longo do projeto. Um dado para corroborar esta complexidade se traduz nas mais de cem atas de reuniões formais documentadas.

Para ilustrar alguns momentos em que a troca de experiências e a aprendizagem dos membros da equipe foram marcantes, podemos destacar: (a) as discussões sobre a Taxonomia de Bloom, desconhecida pela maioria dos colaboradores da área da Computação; (b) a assimilação dos conceitos de competência e habilidade, cuja clareza na diferenciação entre ambos mostrou-se uma tarefa árdua para a equipe de Computação; (c) a validação de modelos funcionais (casos de uso) e de dados (classes de domínio) pela equipe da área da Educação. Embora novos, a compreensão desses modelos pelo grupo da Educação foi facilitada em função da experiência prévia de alguns componentes na manipulação de mapas conceituais cuja estrutura em muito se assemelha aos referidos modelos.

O segundo macro-passo da metodologia de desenvolvimento é a sensibilização do corpo social das duas escolas. Para este passo, foi estruturado um planejamento em conjunto com a equipe da SME. Fizeram parte do planejamento agendas com o detalhamento, no formato de passo a passo, do que iria ocorrer em cada encontro. Os encontros foram realizados separadamente. Sendo o primeiro com os gestores, depois docentes, discentes e, por último, com os responsáveis pelas crianças. Para cada grupo, foi preparado um material específico. Buscou-se, em conjunto com a equipe da SME, desenvolver o material que os interlocutores pudessem ter total compreensão do MEMORE.

Para as etapas de elicitação de requisitos, validação, modelagem e o desenvolvimento dos protótipos das funcionalidades como também para as reuniões de validação dos protótipos foi adotado o Práxis. O Práxis, no que diz respeito aos aspectos técnicos, é um processo de desenvolvimento de software que detalha com métodos, boas práticas e técnicas o que precisa ser feito, como será feito, quem irá fazer, quanto será feito, quais insumos irá utilizar e quais resultados irá produzir [Paula Filho 2005].

Sessões de observação de aulas utilizando os laptops também foram necessárias ao longo do processo. Todo o desenvolvimento do ambiente computacional proposto foi baseado nos princípios da orientação a objetos. As equipes gestoras das duas escolas piloto tiveram papel fundamental na elaboração dos modelos, nos testes e na validação do protótipo.

Com relação aos dois últimos macro-passos, a implantação e homologação, ambos foram agendados para outubro de 2012. No entanto, o desenvolvimento do ambiente MEMORE está bastante avançado. O módulo III de Descoberta de Conhecimento, por exemplo, está todo implementado. Neste momento, estamos realizando um conjunto, bastante diversificado, de teste de caixa branca e preta (Paula 
Filho, 2005). Esses testes estão apontando para uma maturidade e robustez do módulo, que está praticamente sem erros a serem corrigidos.

Uma solução, protótipo e experimental, do agente de coleta de dados, foi instalada nos laptops das crianças das turmas piloto, na E. M. Rosa Carelli da Costa. Esta versão experimental está, há mais de trinta dias, realizando o monitoramento de uso de alguns aplicativos indicados para os testes preliminares desse agente. Já o módulo de Transferência de Dados está com a versão beta pronta e em fase de testes há mais de quinze dias nos dispositivos das turmas piloto. Detalhes sobre os resultados dos experimentos podem ser obtidos em Goldschmidt et al. (2012).

Atualmente a equipe do projeto está dedicada ao desenvolvimento do portal MEMORE. Entre as principais funcionalidades deste portal, está o conjunto de cadastros básicos. Eles permitem a inicialização de todo o ambiente a partir das informações essenciais ao seu funcionamento, tais como, os cadastros dos usuários, escolas, níveis de acesso, dispositivos, entre outros. Os relatórios gerenciais, o controle de concessões de dispositivos e a comunidade de prática de projetos de aprendizagem estão entre as funcionalidades elencadas como de grande relevância e utilidade tanto para as escolas quanto para o PROUCA.

\section{Resultados indiretos e subprodutos do projeto}

As escolas passaram a conviver com as práticas da pesquisa, da escrita de artigos, escrita de relatórios, entre outros. Este movimento da universidade interagindo todas as semanas com as escolas alterou a autoestima dos professores. Dois deles procuraram a universidade e solicitaram apoio para fazer o mestrado em Educação. Mencionaram o interesse de desenvolver a dissertação a partir de alguma demanda do MEMORE. Outra professora ligada à SME solicitou o mesmo apoio, só que para cursar o doutorado.

Outra oportunidade de aprendizagem foi gerada pelo desafio do projeto MEMORE lidar com uma equipe muito numerosa. Nesse sentido, a criação e a utilização de diversos artefatos de documentação e controle foram essenciais. Vários tipos de formulários tais como modelo de ata de reunião, fichas de cadastro de colaboradores e de relato de ocorrências de teste, modelos de relatório de atividades semanais (para preenchimento pelos bolsistas), de observação (do uso da tecnologia em sala de aula), e de relatório técnico foram concebidos no início do projeto e aplicados amplamente ao longo de sua execução.

A extensão do projeto MEMORE e sua consequente complexidade também pode ser aferida pelos artefatos de software gerados. Como esperado em um processo de desenvolvimento de sistema computacional, vários artefatos foram sendo produzidos durante o projeto. Um modelo de casos de uso, seis modelos de classes de domínio, sessenta e seis tabelas em um banco de dados relacional, mais de cem interfaces homem-computador e outras centenas de unidades de programação, totalizando mais de trinta mil linhas de código. Enfim, tais números ilustram a real dimensão e a dificuldade de gerenciar todo o ambiente que está sendo desenvolvido.

Uma das primeiras contribuições do MEMORE foi a elaboração de um site para socialização de informações sobre objetivos, metodologia, ideias, ações, notícias e 
produtos gerados ${ }^{2}$. Para tanto, foi utilizado o JOOMLA como gerenciador de conteúdo. Alunos e professores da área da Computação se uniram em uma profícua troca de conhecimentos sobre a ferramenta. A utilização de um gerenciador de conteúdos tem proporcionado grande facilidade na constante atualização das informações lá disponibilizadas.

Além disso, apresentações de divulgação do projeto foram construídas para cada público do corpo social da escola. Três pôsteres elaborados, um para os aspectos técnicos do projeto e os outros dois foram projetados sob medida para as escolas piloto do projeto. A equipe do MEMORE também participou, até o presente momento, de dois eventos sobre Tecnologia Educacional onde foi possível apresentar o projeto. Neste período também tivemos a produção de três artigos, um publicado e outros dois em processo de avaliação.

A proposta original do MEMORE, essencialmente técnica, continha um planejamento para atuação nas escolas que precisou ser adaptado em função das realidades encontradas. A adaptação desse planejamento em função dos tempos e dos espaços escolares foi consequência natural do processo e teve um caráter muito enriquecedor para o grupo da Computação. O conhecimento dos futuros usuários do sistema, mais do que nunca, mostrou-se fundamental não só para a construção do ambiente computacional, mas também para a configuração da metodologia de trabalho. A partir de um cronograma geral, formalmente aprovado junto ao $\mathrm{CNPq}$, desenvolvedores e usuários passaram a elaborar em conjunto um planejamento semanal detalhado com metas e prioridades bem definidas e viáveis. Sempre respeitando os horários das atividades escolares.

\section{Considerações finais}

O projeto UCA e o programa PROUCA carecem de instrumentos que permitam aos gestores educacionais conhecer, acompanhar e avaliar ações pedagógicas vinculadas ao uso dos laptops nas salas de aula e fora delas [CNPq 2010]. Diante desse cenário, este artigo teve como objetivo apresentar o relato de uma experiência de integração universidade-escola na construção coletiva de um sistema de monitoramento do programa um computador por aluno: o MEMORE. O texto também traz observações relevantes para a execução do projeto e lista, em detalhes, os resultados indiretos e os subprodutos oriundos do projeto.

Como trabalho futuro, o conjunto de ações adotados no projeto deverá servir de base para a formalização de uma metodologia de desenvolvimento coletivo de software de gestão para a área da educação.

\section{Referências}

Benitti, F. B. V.; Seara, E. F. R.; Schlindwein, L. M. (2005) Processo de

Desenvolvimento de Software Educacional: proposta e experimentação. Revista Novas Tecnologias na Educação CINTED-UFRGS, Vol. 3, Num. 1.

\footnotetext{
${ }^{2}$ r1.ufrrj.br/im/memore
} 
Burd, L. (1999) Desenvolvimento de software para atividades educacionais. Dissertação de Mestrado. Universidade Estadual de Campinas, Faculdade de Engenharia Elétrica e de Computação. Campinas, SP: [s.n.].

CNPq/CAPES/SEED-MEC (2010) Edital no. 76/2010. PROUCA Fase 2. Disponível: http://memoria.cnpq.br/editais/ct/2010/docs/076.pdf. Acesso: 05 de Julho de 2012.

Goldschmidt, R. R. et al. (2012) MEMORE - Um Ambiente Computacional para Apoio ao Acompanhamento do Programa Um Computador por Aluno, In: Anais do XXIII SBIE- XVIII WIE, SBC, Rio de Janeiro.

Goldschmidt, R. R.; Passos, E. P. L. (2005). Data Mining. Rio de Janeiro: Ed. Campus.

Hansen, N. et al. (2012) Laptop usage affects abstract reasoning of children in the developing world. In: Computers \& Education, n. 59, pages 989-1000. Elsevier.

Lacerda, M. (2012) "Informática como Disciplina Obrigatória na Educação Básica", In: IX Encontro Virtual de Documentação em Software Livre e VI Congresso Internacional de Linguagem e Tecnologia online (Evidosol/Ciltec-online). v.1, n. 1.

Meneses, S. C. P. (2011) UCA - Um Computador por Aluno: Era da Inclusão Digital, In: Anais do XXII SBIE- XVII WIE, SBC, Aracaju. Disponível: http://www.brie.org/sbie-wie2011/SBIE-Trilha7/92966_1.pdf. Acesso: 5 de julho de 2012.

Motta, R. A. S. M., Santos, T. A. C., Goldschmidt, R. R., Campos, M. F. (2009) Escola Mandala em Ação. Rio de Janeiro: Imprinta.

Nugroho, D., Longsdale, M. (2010) Evaluation of OLPC programs globally: a literature review (version 4). Australian Council for Education Research. Disponível: http://wiki.laptop.org/images/a/a5/olpc_Lit_Review_v4_Aug2010.pdf. Acesso: 12 de junho de 2012.

Paula Filho, W. P. (2005) Engenharia de Software. Rio de Janeiro: LTC.

Penuel, W. R. (2006) Implementation and effects of one-to-one computing initiatives. Journal of Research on Technology in Education, n. 38, pages 329-348.

Perry, G. T. (2005) Proposta de uma Metodologia Participativa para o Desenvolvimento de Software Educacional. Dissertação de Mestrado. Programa de Pós-graduação em Engenharia de Produção. Porto Alegre: Escola de Engenharia, UFRGS.

Russell, S. J., Norvig, P. (2004) Inteligência Artificial. 2a . ed. Rio de Janeiro: Campus.

Santos, F.V.; Almeida, J. F.; Nassar, A. B.; Bassalo, J.M.F.; Souza Sobrinho, C.L.S. (2012) A Interação Tecnológica no Modelo Pedagógico da Educação Básica Brasileira, In: Anais do DEsafIE! - I Workshop de Desafios da Computação Aplicada à Educação. CSBC2012. Curitiba/PR.

Tajra, S. F. (2008) Informática na Educação, São Paulo: Érica.

\section{Apoio}

Este trabalho recebe fomento do Conselho Nacional de Pesquisa (CNPq) sob o processo número: 550370/2011-0. O Centro de Tecnologia da Informação e Comunicação do Estado do Rio de Janeiro (PRODERJ) é responsável pela hospedagem gratuita dos servidores do projeto. 